\title{
An Overlapping Schwarz Method for Singularly Perturbed Fourth-Order Convection-Diffusion Type
}

\author{
J. Christy Roja ${ }^{a}$ and Ayyadurai Tamilselvan ${ }^{b}$ \\ ${ }^{a}$ Department of Mathematics, St.Joseph's College \\ Tiruchirappalli-620 002, Tamilnadu, India \\ ${ }^{b}$ Department of Mathematics, Bharathidasan University \\ Tiruchirappalli-620 024, Tamilnadu, India \\ E-mail(corresp.): jchristyrojaa@gmail.com \\ E-mail: mathats@bdu.ac.in
}

Received June 13, 2019; revised August 31, 2020; accepted August 31, 2020

\begin{abstract}
In this paper, we have constructed an iterative numerical method based on an overlapping Schwarz procedure with uniform mesh for singularly perturbed fourth-order of convection-diffusion type. The method splits the original domain into two overlapping subdomains. A hybrid difference scheme is proposed in which on the boundary layer region we use the central finite difference scheme on a uniform mesh while on the non-layer region we use the mid-point difference scheme on a uniform mesh. It is shown that the method produces numerical approximations which converge in the maximum norm to the exact solution. We prove that, when appropriate subdomains are used the method produces convergence of almost second-order. Furthermore, it is shown that, two iterations are sufficient to achieve the expected accuracy. Numerical examples are presented to support the theoretical results.
\end{abstract}

Keywords: singularly perturbed problems, convection-diffusion equations, Schwarz method, hybrid difference scheme.

AMS Subject Classification: 65L10.

\section{Introduction}

Singular Perturbation Problems (SPPs) appear in many branches of applied mathematics, like fluid dynamics, quantum mechanics, turbulent interaction of waves and currents, electrodes theory, etc. The convergence of the numerical approximations generated by standard numerical methods applied to such 
problems depends adversely on the singular perturbation parameter. Most of these works have concentrated on second-order single differential equations ( [4] and the references therein), but for fourth-order equations only few results are reported in the literature $[2,15,16,17]$.

Numerical methods for singularly perturbed problems comprising domain decomposition and Schwarz iterative techniques have been examined by various authors, for example, in $[1,6,7,8,9,10,18,20]$. In [10], the authors examined a continuous overlapping Schwarz method for a singularly perturbed convectiondiffusion equation with arbitrary fixed interface positions and found it to be uniformly convergent with respect to the perturbation parameter. In [20], an analysis of overlapping domain decomposition methods for singularly perturbed reaction-diffusion problems with distinct small positive parameters is presented. The authors of [20] found a flaw in the analysis of domain decomposition methods explored in $[6,13,18]$. The authors observation is that the constant $C$ is not independent of the iteration number $k$ and it is growing at each induction step in their proof of $[6,13,18]$. But in $[20]$ the authors have presented an alternate analysis of overlapping domain decomposition methods for singularly perturbed reaction-diffusion problems with two parameters and problems in [18].

The authours of $[8,9]$ have concluded that the numerical solution of classical finite difference scheme used in Schwarz method does not converge to the exact solution of their test problem which is a single equation. But our proposed scheme used in Schwarz method [3] has overcome the fundamental difficulty mentioned by the authours of $[8,9]$. In $[8,9]$, the authors used the same scheme in both the layer and non-layer regions, whereas in our case we used different schemes in each region.

As far as the authors knowledge goes fourth-order SPPs have not yet been examined for higher-order of convergence. Therefore, we are interested in constructing a numerical method for fourth-order SPPs. Of primary interest we have been proved that when appropriate subdomains are used the method produce convergence of almost second-order.

Motivated by the works of $[2,10,15,16,17]$ we have examined experimentally the performance of Schwarz method to the fourth-order Singularly Perturbed Boundary Value Problems (SPBVPs) described as below.

$$
\begin{gathered}
-\varepsilon y^{i v}(x)+a(x) y^{\prime \prime \prime}(x)+b(x) y^{\prime \prime}(x)-c(x) y(x)=-f(x), \quad x \in \Omega, \\
y(0)=q_{1}, \quad y^{\prime \prime}(0)=-q_{2}, \quad y(1)=q_{3} \quad y^{\prime \prime}(1)=-q_{4},
\end{gathered}
$$

where $a(x), b(x), c(x)$ are sufficiently smooth functions satisfying the following conditions:

$$
\begin{array}{r}
a(x) \geq \alpha, \quad \alpha>1, \\
b(x) \geq 0 \\
0 \geq c(x) \geq-\gamma, \quad \gamma>0 \\
\alpha>5 \gamma
\end{array}
$$

with $0<\varepsilon \ll 1, \Omega=(0,1), \quad \bar{\Omega}=[0,1] \quad$ and $y \in C^{(4)}(\Omega) \cap C^{(2)}(\bar{\Omega})$, which have important applications in fluid dynamics, have been studied in [5], and 
the references therein. The SPBVPs (1.1)-(1.2) can be transformed into an equivalent weakly coupled system of two ODEs subject to suitable boundary conditions of the form:

$$
\begin{aligned}
& \left\{\begin{array}{l}
L_{1} \boldsymbol{y}(x) \equiv-y_{1}^{\prime \prime}(x)-y_{2}(x)=0, \quad x \in \Omega, \\
L_{2} \boldsymbol{y}(x) \equiv-\varepsilon y_{2}^{\prime \prime}(x)+a(x) y_{2}^{\prime}(x) \\
+b(x) y_{2}(x)+c(x) y_{1}(x)=f(x), \quad x \in \Omega,
\end{array}\right. \\
& y_{1}(0)=q_{1}, \quad y_{2}(0)=q_{2}, \quad y_{1}(1)=q_{3}, \quad y_{2}(1)=q_{4},
\end{aligned}
$$

where $\boldsymbol{y}=\left(y_{1}, y_{2}\right)^{T}$ and $a(x), b(x), c(x)$ are sufficiently smooth functions satisfying (1.3)-(1.5). The above weakly coupled system can be written in the matrix-vector form as

$$
\begin{aligned}
& \mathbf{L} \boldsymbol{y} \equiv\left(\begin{array}{l}
L_{1} \boldsymbol{y} \\
L_{2} \boldsymbol{y}
\end{array}\right) \equiv\left(\begin{array}{cc}
-\frac{d^{2}}{d x^{2}} & 0 \\
0 & -\varepsilon \frac{d^{2}}{d x^{2}}
\end{array}\right) \boldsymbol{y}+\mathbf{A}(x) \boldsymbol{y}^{\prime}+\mathbf{B}(x) \boldsymbol{y}=\mathbf{f}(x), x \in \Omega, \\
& \boldsymbol{y}(0)=\left(\begin{array}{l}
q_{1} \\
q_{2}
\end{array}\right), \quad \boldsymbol{y}(1)=\left(\begin{array}{l}
q_{3} \\
q_{4}
\end{array}\right),
\end{aligned}
$$

where $\boldsymbol{y}(x)=\left(\begin{array}{l}y_{1}(x) \\ y_{2}(x)\end{array}\right), \quad \mathbf{f}(x)=\left(\begin{array}{c}0 \\ f(x)\end{array}\right), \quad \mathbf{A}(x)=\left(\begin{array}{cc}0 & 0 \\ 0 & a(x)\end{array}\right)$ and $\mathbf{B}(x)=$ $\left(\begin{array}{cc}0 & -1 \\ c(x) & b(x)\end{array}\right)$. Let $\beta=\min \{-1, b(x)+c(x)\}$.

In this paper, of primary interest we have proved that discrete Schwarz method converge to the solution of the continuous problem. The method is shown to be of almost second-order convergence. Furthermore, we show that, just two iterations are required to achieve the expected accuracy.

Remark 1. The solution of the problem (1.1)-(1.2) exhibits a boundary layer at $x=1$ which is less severe because the boundary conditions are prescribed for the derivative of the solution [14]. The condition (1.3) says that (1.1)-(1.2) is a non-turning point problem. The condition (1.4) is known as the quasimonotonicity condition [14]. The maximum principle theorem for the above system (1.1)-(1.2) and for the corresponding discrete problem are established using the conditions (1.3)-(1.4) and using this principle, we can establish a stability result.

The outline of rest of the paper is as follows. In Section 2, the continuous Schwarz method is described. The derivative estimates are obtained in Section 3. In Section 4, the discrete Schwarz method is described. The maximum pointwise error bounds are obtained in Section 5. Numerical experiments are presented in Section 6 and finally, conclusions are included in Section 7.

Notations: Throughout the paper we use $C$, with or without subscript to denote a generic positive constant independent of $\varepsilon$, the iteration $k$ and the discretization parameter $N$.

Let $\boldsymbol{y}: D \rightarrow \mathbb{R}, D \subseteq \mathbb{R}$. The appropriate norm for studying the convergence of the numerical solution to the exact solution of a SPP is $\|y\|_{D}=\sup _{x \in D}|y(x)|$. For a vector $\boldsymbol{y}=\left(y_{1}, y_{2}\right)^{T}$, we define $\|\boldsymbol{y}\|=\max _{j=1,2}\left|y_{j}\right|$. 
For a vector valued function $\boldsymbol{z}=\left(z_{1}, z_{2}\right)^{T}$, define $\|\boldsymbol{z}\|_{\Omega}=\max \left\{\left\|z_{1}\right\|_{\Omega},\left\|z_{2}\right\|_{\Omega}\right\}$. Given any two vector valued functions, $\boldsymbol{z}$ and $\boldsymbol{y}, \boldsymbol{z} \geq \boldsymbol{y}$ if $z_{j} \geq y_{j}$ for all $j=1,2$. For a vector of mesh functions $\mathbf{Z}\left(x_{i}\right)=\left(Z_{1}\left(x_{i}\right), Z_{2}\left(x_{i}\right)\right)^{T}$, define

$$
\|Z\|_{\Omega^{N}}=\max _{j=1,2}\left(\max _{x_{i} \in \Omega^{N}}\left|Z_{j}\left(x_{i}\right)\right|\right) .
$$

\section{Continuous Schwarz method}

In this section, a continuous Schwarz method is described. This process generates a sequence of iterates $\left\{\boldsymbol{y}^{[k]}\right\}$, which converges as $k \rightarrow \infty$ to the exact solution $\boldsymbol{y}$. Further we prove the maximum principle for (1.6)-(1.7). Using this principle, a stability result is stated. First, we split the domain into two overlapping subdomains as $\Omega_{c}=(0,1-\tau)$ and $\Omega_{r}=(1-2 \tau, 1)$, where the subdomain transition parameter is an appropriate constant, defined in Section 4. The iterative process is defined as follows:

$$
\boldsymbol{y}^{[0]}(x) \equiv 0, \quad 0<x<1, \quad \boldsymbol{y}^{[0]}(0)=\boldsymbol{y}(0), \quad \boldsymbol{y}^{[0]}(1)=\boldsymbol{y}(1) .
$$

For $k \geq 1$, the iterates $\boldsymbol{y}^{[k]}(x)$ are defined by

$$
\boldsymbol{y}^{[k]}(x)=\left\{\begin{array}{lll}
\boldsymbol{y}_{c}^{[k]}(x) & \text { for } & x \in \bar{\Omega}_{c}, \\
\boldsymbol{y}_{r}^{[k]}(x) & \text { for } & x \in \bar{\Omega}_{r} \backslash \bar{\Omega}_{c},
\end{array}\right.
$$

where $\boldsymbol{y}_{p}^{[k]}, \quad p=\{c, r\}$ are the solutions of the problems

$$
\begin{aligned}
& \mathbf{L} \boldsymbol{y}_{r}^{[k]}(x)=\mathbf{f} \text { in } \Omega_{r}, \quad \boldsymbol{y}_{r}^{[k]}(1-2 \tau)=\boldsymbol{y}^{[k-1]}(1-2 \tau), \quad \boldsymbol{y}_{r}^{[k]}(1)=\boldsymbol{y}(1) \\
& \mathbf{L} \boldsymbol{y}_{c}^{[k]}(x)=\mathbf{f} \text { in } \Omega_{c}, \quad \boldsymbol{y}_{c}^{[k]}(0)=\boldsymbol{y}(0), \quad \boldsymbol{y}_{c}^{[k]}(1-\tau)=\boldsymbol{y}_{r}^{[k]}(1-\tau) .
\end{aligned}
$$

Letting $\Omega_{p}=(d, e), \quad \bar{\Omega}_{p}=[d, e], \quad p=\{c, r\}$, note that the BVPs $(1.6)-(1.7)$ satisfies the following maximum principle on each $\bar{\Omega}_{p}$.

Theorem 1. (Maximum principle). Consider the BVPs (1.6)-(1.7). Let $y_{1}(d) \geq 0, \quad y_{2}(d) \geq 0$, and $y_{1}(e) \geq 0$ and $y_{2}(e) \geq 0, L_{1} \boldsymbol{y}(x) \geq 0$, and $L_{2} \boldsymbol{y}(x) \geq 0$, for $x \in \Omega_{p}$. Then, $\boldsymbol{y}(x) \geq 0, \forall x \in \bar{\Omega}_{p}$.

Proof. Define the test functions $s(x)=\left(s_{1}(x), s_{2}(x)\right)^{T}$ by

$$
s_{1}(x)=5-x^{2}, \quad s_{2}(x)=1+x, \quad x \in \bar{\Omega}_{p} .
$$

Clearly, $s_{1}(d)>0, s_{2}(d)>0, s_{1}(e)>0, s_{2}(e)>0$. We can easily prove that $L_{1} s(x)>0$ and $L_{2} s(x)>0$, for $x \in \Omega_{p}$. Assume that the theorem is not true. We define

$$
\xi=\max \left\{\max _{x \in \bar{\Omega}_{p}}\left(-y_{1} / s_{1}\right)(x), \max _{x \in \bar{\Omega}_{p}}\left(-y_{2} / s_{2}\right)(x)\right\} .
$$

Then, $\xi>0$. Also, $\left(y_{1}+\xi s_{1}\right)(x) \geq 0,\left(y_{2}+\xi s_{2}\right)(x) \geq 0, \forall x \in \bar{\Omega}_{p}$. Furthermore, there exists a point $x_{0} \in \Omega_{p}$ such that either

$$
\left(y_{1}+\xi s_{1}\right)\left(x_{0}\right)=0 \quad \text { or } \quad\left(y_{2}+\xi s_{2}\right)\left(x_{0}\right)=0 \quad \text { or both. }
$$


Case 1: $\left(y_{1}+\xi s_{1}\right)\left(x_{0}\right)=0$, for $x_{0} \in \Omega_{p}$. This implies that $y_{1}+\xi s_{1}$ attains its minimum at $x=x_{0}$. Therefore,

$$
0<L_{1}(\boldsymbol{y}+\xi \boldsymbol{s})\left(x_{0}\right)=-\left(y_{1}+\xi s_{1}\right)^{\prime \prime}\left(x_{0}\right)-\left(y_{2}+\xi s_{2}\right)\left(x_{0}\right) \leq 0,
$$

which is a contradiction.

Case 2: $\left(y_{2}+\xi s_{2}\right)\left(x_{0}\right)=0$, for $x_{0} \in \Omega_{p}$. This implies that $y_{2}+\xi s_{2}$ attains its minimum at $x=x_{0}$. Therefore,

$$
\begin{aligned}
0<L_{2}(\boldsymbol{y}+\xi s)\left(x_{0}\right)= & -\varepsilon\left(y_{2}+\xi s_{2}\right)^{\prime \prime}\left(x_{0}\right)+a(x)\left(y_{2}+\xi s_{2}\right)^{\prime}\left(x_{0}\right) \\
& +b(x)\left(y_{2}+\xi s_{2}\right)\left(x_{0}\right)+c(x)\left(y_{1}+\xi s_{1}\right)\left(x_{0}\right) \leq 0,
\end{aligned}
$$

which is a contradiction. Hence it can be conclude that $\boldsymbol{y}(x) \geq 0, \forall x \in \bar{\Omega}$.

An immediate consequence of this is the following stability result.

Lemma 1. (Stability result). If $\boldsymbol{y}(x)$ is the solution of the BVPs (1.6)-(1.7) then $\forall x \in \bar{\Omega}_{p}$

$$
\|\boldsymbol{y}\| \leq C \max \left\{\left|y_{1}(d)\right|,\left|y_{2}(d)\right|,\left|y_{1}(e)\right|,\left|y_{2}(e)\right|, \max _{x \in \Omega_{p}}\left|L_{1} \boldsymbol{y}(x)\right|, \max _{x \in \Omega_{p}}\left|L_{2} \boldsymbol{y}(x)\right|\right\} .
$$

Proof. Set

$$
M=C \max \left\{\left|y_{1}(d)\right|,\left|y_{2}(d)\right|,\left|y_{1}(e)\right|,\left|y_{2}(e)\right|, \max _{x \in \Omega_{p}}\left|L_{1} \boldsymbol{y}(x)\right|, \max _{x \in \Omega_{p}}\left|L_{2} \boldsymbol{y}(x)\right|\right\} .
$$

Define two barrier functions $\boldsymbol{w}^{ \pm}(x)=\left(w_{1}^{ \pm}(x), w_{2}^{ \pm}(x)\right)^{T}$ by

$$
w_{1}^{ \pm}(x)=M\left(5-x^{2}\right) \pm y_{1}(x) \quad \text { and } \quad w_{2}^{ \pm}(x)=M(1+x) .
$$

For $x \in \Omega_{c}$, we have

$$
\begin{aligned}
L_{1} \boldsymbol{w}^{ \pm}(x) & =-w_{1}^{ \pm \prime \prime}(x)-w_{2}^{ \pm}(x)>M \tau \pm L_{1} \boldsymbol{y}(x) \geq 0, \\
L_{2} \boldsymbol{w}^{ \pm}(x) & =-\varepsilon w_{2}^{ \pm \prime \prime}(x)+a(x) w_{2}^{ \pm \prime}(x)+b(x) w_{2}^{ \pm}(x)+c(x) w_{1}^{ \pm}(x), \\
& >M(\alpha-5 \gamma) \pm L_{2} \boldsymbol{y}(x) \geq 0,
\end{aligned}
$$

by a proper choice of $C$. For $x \in \Omega_{r}$, we have

$$
\begin{aligned}
L_{1} \boldsymbol{w}^{ \pm}(x) & =-w_{1}^{ \pm \prime \prime}(x)-w_{2}^{ \pm}(x)=M(1-x) \pm L_{1} \boldsymbol{y}(x) \geq 0, \\
L_{2} \boldsymbol{w}^{ \pm}(x) & =-\varepsilon w_{2}^{ \pm \prime \prime}(x)+a(x) w_{2}^{ \pm \prime}(x)+b(x) w_{2}^{ \pm}(x)+c(x) w_{1}^{ \pm}(x), \\
& >M(\alpha-5 \gamma) \pm L_{2} \boldsymbol{y}(x) \geq 0,
\end{aligned}
$$

by a proper choice of $C$. Furthermore, we have

$$
\begin{aligned}
& w_{1}^{ \pm}(d)=w_{1}^{ \pm}(0)=5 M \pm y_{1}(0) \geq 0, \quad w_{2}^{ \pm}(d)=w_{2}^{ \pm}(0)=M \pm y_{2}(0) \geq 0, \\
& w_{1}^{ \pm}(e)=w_{1}^{ \pm}(1-\tau)>3 M \pm y_{1}(1-\tau) \geq 0, \\
& w_{2}^{ \pm}(e)=w_{2}^{ \pm}(1-\tau)>M \pm y_{2}(1-\tau) \geq 0, \\
& w_{1}^{ \pm}(d)=w_{1}^{ \pm}(1-2 \tau)>4 M \pm y_{1}(1-2 \tau) \geq 0, \\
& w_{2}^{ \pm}(d)=w_{2}^{ \pm}(1-2 \tau)>M \pm y_{2}(1-2 \tau) \geq 0, \\
& w_{1}^{ \pm}(e)=w_{1}^{ \pm}(1)=4 M \pm y_{1}(1) \geq 0, \quad w_{2}^{ \pm}(e)=w_{2}^{ \pm}(1)=2 M \pm y_{2}(1) \geq 0
\end{aligned}
$$

by a proper choice of $C$. Applying Theorem 1 to the barrier functions $\boldsymbol{w}^{ \pm}(x)$, we get the desired result. 


\section{$3 \quad$ Estimates of derivatives}

In Section 5 we establish the convergence of the discrete Schwarz method described in Section 4. To prove convergence of the numerical solution, we need the following stronger results on the estimates of the derivatives of the components of the solution of the BVPs (1.6)-(1.7). Now, decompose the solution $\boldsymbol{y}(x)$ of (1.6)-(1.7) into smooth and singular components $\boldsymbol{v}(x)$ and $\boldsymbol{w}(x)$ respectively as

$$
\boldsymbol{y}(x)=\boldsymbol{v}(x)+\boldsymbol{w}(x),
$$

where $\boldsymbol{v}(x)=\left(v_{1}(x), v_{2}(x)\right)^{T}$ is the solution of the reduced problem of the BVPs (1.6)-(1.7) given by

$$
\left\{\begin{array}{l}
-v_{1}^{\prime \prime}(x)-v_{2}(x)=0 \\
a(x) v_{2}^{\prime}(x)+b(x) v_{2}(x)+c(x) v_{1}(x)=f(x) \\
v_{1}(0)=q_{1}, \quad v_{1}(1)=q_{3}, \quad v_{2}(0)=q_{2}
\end{array}\right.
$$

and $\boldsymbol{w}(x)=\left(w_{1}(x), w_{2}(x)\right)^{T}$ is a layer correction term given by

$$
\left\{\begin{array}{l}
w_{1}(x)=-(\varepsilon / a(0))^{2}\left(q_{4}-v_{2}(1)\right) e^{-a(0)(1-x) / \varepsilon} \\
w_{2}(x)=\left(q_{4}-v_{2}(1)\right) e^{-a(0)(1-x) / \varepsilon}
\end{array}\right.
$$

and $\boldsymbol{w}(\boldsymbol{x})$ satisfies

$$
\left\{\begin{array}{l}
-w_{1}^{\prime \prime}(x)-w_{2}(x)=0, \\
-\varepsilon w_{2}^{\prime \prime}(x)+a(0) w_{2}^{\prime}(x)=0 \\
w_{1}(0)=w_{1}(1) e^{-a(0) / \varepsilon}, \quad w_{1}(1)=-w_{2}(1)(\varepsilon / a(0))^{2}, \\
w_{2}(0)=w_{2}(1) e^{-a(0) / \varepsilon}, \quad w_{2}(1)=q_{4}-v_{2}(1) .
\end{array}\right.
$$

The following lemma gives estimates of the derivatives of these components.

Lemma 2. The solution $\boldsymbol{y}(x)$ of the BVPs (1.6)-(1.7) has the decomposition $\boldsymbol{y}(x)=\boldsymbol{v}(x)+\boldsymbol{w}(x)$ into smooth and singular components, satisfy

$$
\begin{aligned}
& \left|\boldsymbol{v}_{1}^{(l)}(x)\right| \leq C, \quad\left|\boldsymbol{v}_{2}^{(l)}(x)\right| \leq C, \\
& \left|\boldsymbol{w}_{1}^{(l)}(x)\right| \leq C \varepsilon^{-(l-2)} e^{-\alpha(1-x) / \varepsilon}, \quad\left|\boldsymbol{w}_{2}^{(l)}(x)\right| \leq C \varepsilon^{-(l)} e^{-\alpha(1-x) / \varepsilon},
\end{aligned}
$$

for $0 \leq l \leq 4, \forall x \in \bar{\Omega}=\left(\bar{\Omega}_{r} \backslash \bar{\Omega}_{c}\right) \cup \bar{\Omega}_{c}, \boldsymbol{v}(x)$ and $\boldsymbol{w}(x)$ are given by (3.2)-(3.3).

Proof. It is easy to check that

$$
\left|v_{1}^{(l)}(x)\right| \leq C \quad \text { and } \quad\left|v_{2}^{(l)}(x)\right| \leq C \quad \text { for } \quad x \in \bar{\Omega}
$$

as $a(x), b(x), c(x), f(x)$ are sufficiently smooth functions. Differentiating the equation (3.3) $l$ times and using the method of induction one can get

$$
\begin{aligned}
& \left|w_{1}^{(l)}(x)\right| \leq C \varepsilon^{-(l-2)} \exp (-\alpha(1-x) / \varepsilon), \\
& \left|w_{2}^{(l)}(x)\right| \leq C \varepsilon^{-(l)} \exp (-\alpha(1-x) / \varepsilon) .
\end{aligned}
$$




\section{Discrete Schwarz method}

The continuous overlapping Schwarz method described in Section 2 is discretized by introducing uniform meshes on each subdomain. The domain $\Omega=$ $(0,1)$ is divided into two overlapping subdomains as $\Omega_{c}=(0,1-\tau)$ and $\Omega_{r}=$ $(1-2 \tau, 1)$. The subdomain transition parameter $\tau$ is chosen to be the Shishkin transition point $\tau=\min \left\{\frac{1}{3}, \frac{4 \varepsilon}{\alpha} \ln N\right\}$ as in ([10], p.91). In each subdomain, $\Omega_{p}=(d, e), \quad p=\{c, r\}$, construct a uniform mesh $\bar{\Omega}_{p}^{N}=\left\{d=x_{0}<x_{1}<\right.$ $\left.x_{2}<\cdots<x_{n}=e\right\}$ with $h_{p}=\left(x_{i}-x_{i-1}\right) / N=(e-d) / N$.

In the proposed scheme we use the central finite difference scheme with a uniform mesh on the subdomain $\Omega_{r}$ and the mid-point difference scheme with a uniform mesh on the subdomain $\Omega_{c}$. Then in each subdomain $\Omega_{p}^{N}, p=\{c, r\}$, the corresponding discretization is,

$$
\begin{gathered}
\mathbf{L}^{N} \mathbf{Y}_{c}\left(x_{i}\right)=\left\{\begin{array}{r}
L_{1}^{N} \mathbf{Y}_{c}\left(x_{i}\right)=-\delta^{2} Y_{1, c}\left(x_{i}\right)-\hat{Y}_{2, c}\left(x_{i}\right)=0, \quad i=1, \ldots, N-1, \\
L_{2}^{N} \mathbf{Y}_{c}\left(x_{i}\right)=-\varepsilon \delta^{2} Y_{2, c}\left(x_{i}\right)+a_{i-1 / 2} D^{-} Y_{2, c}\left(x_{i}\right)+c_{i-1 / 2} \hat{Y}_{1, c}\left(x_{i}\right) \\
+b_{i-1 / 2} \hat{Y}_{2, c}\left(x_{i}\right)=f_{i-1 / 2}, \quad i=1, \ldots, N-1,
\end{array}\right. \\
\mathbf{L}^{N} \mathbf{Y}_{r}\left(x_{i}\right)=\left\{\begin{array}{r}
L_{1}^{N} \mathbf{Y}_{r}\left(x_{i}\right)=-\delta^{2} Y_{1, r}\left(x_{i}\right)-Y_{2, r}\left(x_{i}\right)=0, \quad i=1, \ldots, N-1, \\
L_{2}^{N} \mathbf{Y}_{r}\left(x_{i}\right)=-\varepsilon \delta^{2} Y_{2, r}\left(x_{i}\right)+a_{i} D^{0} Y_{2, r}\left(x_{i}\right)+b_{i} Y_{2, r}\left(x_{i}\right) \\
+c_{i} Y_{1, r}\left(x_{i}\right)=f_{i}, \quad i=1, \ldots, N-1,
\end{array}\right.
\end{gathered}
$$

where

$$
\begin{aligned}
& \delta^{2} Y_{j, p}\left(x_{i}\right)=\frac{1}{h_{p}^{2}}\left(Y_{j, p}\left(x_{i+1}\right)-2 Y_{j, p}\left(x_{i}\right)+Y_{j, p}\left(x_{i-1}\right)\right), \\
& D^{-} Y_{j, c}\left(x_{i}\right)=\frac{Y_{j, c}\left(x_{i}\right)-Y_{j, c}\left(x_{i-1}\right)}{h_{c}}, \quad \hat{Y}_{j, c}\left(x_{i}\right) \equiv\left(Y_{j, c}\left(x_{i}\right)+Y_{j, c}\left(x_{i-1}\right)\right) / 2, \\
& D^{0} Y_{j, r}\left(x_{i}\right)=\frac{Y_{j, r}\left(x_{i+1}\right)-Y_{j, r}\left(x_{i-1}\right)}{2 h_{r}}, a_{i-1 / 2} \equiv a\left(\left(x_{i-1}+x_{i}\right) / 2\right), a_{i} \equiv a\left(x_{i}\right),
\end{aligned}
$$

similarly for $b_{i-1 / 2}, c_{i-1 / 2}, f_{i-1 / 2}, b_{i}, c_{i}$ and $f_{i}, j=1,2$.

The discrete problem is $\mathbf{L}^{N} \mathbf{Y}_{p}\left(x_{i}\right)=\mathbf{f}\left(x_{i}\right)$, where

$$
\mathbf{f}\left(x_{i}\right)=\left\{\begin{array}{l}
\mathbf{f}_{i-\frac{1}{2}}, \quad x_{i} \in \bar{\Omega}_{c}^{N}, \\
\mathbf{f}_{i}, \quad x_{i} \in \bar{\Omega}_{r}^{N} .
\end{array}\right.
$$

Then the algorithm for discrete Schwarz method is defined as follows.

Step1: We choose the initial mesh function

$$
\mathbf{Y}^{[0]}\left(x_{i}\right) \equiv 0, \quad 0<x_{i}<1, \quad \mathbf{Y}^{[0]}(0)=\boldsymbol{y}(0), \quad \mathbf{Y}^{[0]}(1)=\boldsymbol{y}(1) .
$$

Step2: We compute the mesh functions $\mathbf{Y}_{p}{ }^{[k]}, p=\{r, c\}$ which are the solutions of the following discrete problems

$$
\begin{aligned}
& \mathbf{L}^{N} \mathbf{Y}_{r}^{[k]}\left(x_{i}\right)=\mathbf{f}_{i}, \quad x_{i} \in \Omega_{r}^{N}, \quad \mathbf{Y}_{r}^{[k]}(1-2 \tau)=\overline{\mathbf{Y}}^{[k-1]}(1-2 \tau), \quad \mathbf{Y}_{r}^{[k]}(1)=\boldsymbol{y}(1), \\
& \mathbf{L}^{N} \mathbf{Y}_{c}^{[k]}\left(x_{i}\right)=\mathbf{f}_{i-\frac{1}{2}}, \quad x_{i} \in \Omega_{c}^{N}, \quad \mathbf{Y}_{c}^{[k]}(0)=\boldsymbol{y}(0), \quad \mathbf{Y}_{c}^{[k]}(1-\tau)=\overline{\mathbf{Y}}_{r}^{[k]}(1-\tau),
\end{aligned}
$$


where $\overline{\boldsymbol{Y}}^{[k]}, k \geq 1$ denotes the piecewise linear interpolant of $\boldsymbol{Y}^{[k]}$ on the mesh $\bar{\Omega}^{N}:=\left(\bar{\Omega}_{r}^{N} \backslash \bar{\Omega}_{c}\right) \cup \bar{\Omega}_{c}^{N}$.

Step3: We compute the mesh function $\mathbf{Y}^{[k]}$ by combining together the solutions on the subdomains

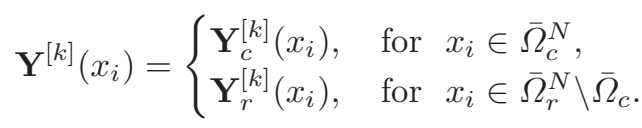

Step4: If the stopping criterion $\left\|\mathbf{Y}^{[k+1]}-\mathbf{Y}^{[k]}\right\|_{\bar{\Omega}^{N}} \leq t o l$ is reached, then stop; otherwise go to Step 2. Here tol is the user prescribed accuracy. For each $p=\{c, r\}$, the matrix associated with $\mathbf{L}^{N}$ is M-matrix, and hence it satisfies the following discrete maximum principle.

Lemma 3. (Discrete maximum principle) Assume that $\boldsymbol{Y}\left(x_{0}\right) \geq \boldsymbol{O}$ and $\boldsymbol{Y}\left(x_{N}\right) \geq \boldsymbol{0}$, then $\boldsymbol{L}^{N} \boldsymbol{Y}\left(x_{i}\right) \geq \boldsymbol{0}, \forall x_{i} \in \Omega_{p}^{N}$ implies that $\boldsymbol{Y}\left(x_{i}\right) \geq \boldsymbol{0}, \forall x_{i} \in \bar{\Omega}_{p}^{N}$.

Proof. Please refer to $[11,12]$ and [19].

An immediate consequence of this lemma is the following stability result.

Lemma 4. If $Y_{j}\left(x_{i}\right)$ is any mesh function then for all $x_{i} \in \bar{\Omega}_{p}^{N}$

$$
\begin{aligned}
& \left|Y_{j}\left(x_{i}\right)\right| \leq C \max \left\{\left|Y_{1}\left(x_{0}\right)\right|,\left|Y_{1}\left(x_{N}\right)\right|,\left|Y_{2}\left(x_{0}\right)\right|,\left|Y_{2}\left(x_{N}\right)\right|,\left\|L_{1}^{N} \boldsymbol{Y}\right\|_{\Omega_{p}^{N}},\left\|L_{2}^{N} \boldsymbol{Y}\right\|_{\Omega_{p}^{N}}\right\} \\
& \text { for } j=1,2
\end{aligned}
$$

Proof. Please refer to $[11,12]$ and [19].

\section{$5 \quad$ Error estimates}

In this Section, we estimate the error in discrete Schwarz iterates and prove that two iterations are required to attain almost second-order convergence. Following the method of analysis adapted in [18] and [20] we derive error estimates. The analysis proceeds as follows.

Lemma 5. Let $\boldsymbol{y}$ be the solution of (1.6)-(1.7) and let $\boldsymbol{Y}^{[k]}$ be the $k^{\text {th }}$ iterate of the discrete Schwarz method described as in Section 4. Then, there are constants $C$ such that

$$
\left\|\boldsymbol{Y}^{[k]}-\boldsymbol{y}\right\|_{\bar{\Omega}^{N}} \leq C 2^{-k}+C N^{-2} \ln ^{3} N .
$$

Proof. At the first iteration $\left(\mathbf{Y}^{[0]}-\boldsymbol{y}\right)(0)=\mathbf{0}$ and $\left(\mathbf{Y}^{[0]}-\boldsymbol{y}\right)(1)=\mathbf{0}$. Since $\mathbf{Y}^{[0]}\left(x_{i}\right)=\mathbf{0}$ for $x_{i} \in \Omega^{N}:=\left\{x_{1}<x_{2}<x_{3} \cdots<x_{N-1}\right\}$ we can use Lemma 1 to show that

$$
\left\|\mathbf{Y}^{[0]}-\boldsymbol{y}\right\|_{\Omega^{N}}=\|\boldsymbol{y}\|_{\Omega^{N}} \leq C .
$$

Clearly, there are constants $C$ such that

$$
\left\|\mathbf{Y}^{[0]}-\boldsymbol{y}\right\|_{\bar{\Omega}^{N}} \leq C 2^{0}+C N^{-2} \ln ^{3} N .
$$


Thus, the result holds for $k=0$ and the proof is now completed by induction. Assume that, for an arbitrary integer $k \geq 0$, there exists $C$ such that

$$
\left\|\mathbf{Y}^{[k]}-\boldsymbol{y}\right\|_{\bar{\Omega}^{N}} \leq C 2^{-k}+C N^{-2} \ln ^{3} N \text {. }
$$

Case (i): Error bound estimation on $\bar{\Omega}_{r}^{N}$. In the proposed scheme we use the central finite difference scheme on $\bar{\Omega}_{r}^{N}$. One can deduce the following truncation error estimate as in [12] on $x_{i} \in \bar{\Omega}_{r}^{N}$ as

$$
\left\|\mathbf{L}^{N}(\mathbf{Y}-\boldsymbol{y})\right\|_{\Omega_{r}^{N}} \leq\left(\begin{array}{c}
C h_{r}^{2}\left\|y_{1}^{(4)}\right\|_{\Omega_{r}^{N}} \\
C \varepsilon h_{r}^{2}\left\|y_{2}^{(4)}\right\|_{\Omega_{r}^{N}}+C h_{r}^{2}\left\|y_{2}^{(3)}\right\|_{\Omega_{r}^{N}}
\end{array}\right) .
$$

In order to find a bound on $\left\|\mathbf{L}^{N}\left(\mathbf{Y}_{r}^{[k+1]}-\boldsymbol{y}\right)\right\|_{\Omega_{r}^{N}}$ we must decompose $\boldsymbol{y}$ as in (3.1). Consider

$$
\begin{aligned}
\left\|\mathbf{L}^{N}\left(\mathbf{Y}_{r}^{[k+1]}-\boldsymbol{y}\right)\right\|_{\Omega_{r}^{N}} & =\|\mathbf{f}-\mathbf{L} \boldsymbol{y}\|_{\Omega_{r}^{N}}=\left\|\left(\mathbf{L}^{N}-\mathbf{L}\right) \boldsymbol{y}\right\|_{\Omega_{r}^{N}} \\
& \leq\left\|\left(\mathbf{L}^{N}-\mathbf{L}\right) \boldsymbol{v}\right\|_{\Omega_{r}^{N}}+\left\|\left(\mathbf{L}^{N}-\mathbf{L}\right) \boldsymbol{w}\right\|_{\Omega_{r}^{N}} .
\end{aligned}
$$

For the first term on the right-hand side of (5.2), we use the local truncation error estimate (5.1), $h_{r} \leq C N^{-1}, \varepsilon \leq C N^{-1}$, and Lemma 2 to get

$$
\begin{aligned}
\left\|\left(\mathbf{L}^{N}-\mathbf{L}\right) \boldsymbol{v}\right\|_{\Omega_{r}^{N}} & \leq\left(\begin{array}{c}
C h_{r}^{2}\left\|v_{1}^{(4)}\right\|_{\Omega_{r}^{N}} \\
C \varepsilon h_{r}^{2}\left\|v_{2}^{(4)}\right\|_{\Omega_{r}^{N}}+C h_{r}^{2}\left\|v_{2}^{(3)}\right\|_{\Omega_{r}^{N}}
\end{array}\right) \\
& \leq\left(\begin{array}{c}
C N^{-2} \\
C N^{-3}+C N^{-2}
\end{array}\right) \leq C N^{-2}
\end{aligned}
$$

For the second term on the right-hand side of (5.2), when $\tau=\frac{4 \varepsilon}{\alpha} \ln N$, using the local truncation error estimate (5.1), and $h_{r} \leq C \varepsilon N^{-1} \ln N$, we have

$$
\begin{aligned}
\left\|\left(\mathbf{L}^{N}-\mathbf{L}\right) \boldsymbol{w}\right\|_{\Omega_{r}^{N}} & \leq\left(\begin{array}{c}
C h_{r}^{2}\left\|w_{1}^{(4)}\right\|_{\Omega_{r}^{N}} \\
C \varepsilon h_{r}^{2}\left\|w_{2}^{(4)}\right\|_{\Omega_{r}^{N}}+C h_{r}^{2}\left\|w_{2}^{(3)}\right\|_{\Omega_{r}^{N}}
\end{array}\right) \\
& \leq\left(\begin{array}{c}
C h_{r}^{2} \varepsilon^{-2} \\
C h_{r}^{2} \varepsilon^{-3}
\end{array}\right) \leq C \varepsilon^{-1} N^{-2} \ln ^{2} N .
\end{aligned}
$$

Using the above estimates in (5.2), we have

$$
\left\|\mathbf{L}^{N}\left(\mathbf{Y}_{r}^{[k+1]}-\boldsymbol{y}\right)\right\|_{\Omega_{r}^{N}} \leq C N^{-2} \ln ^{3} N+C \varepsilon^{-1} N^{-2} \ln ^{2} N
$$

for some $C$. The end point of the subdomain $\Omega_{r}^{N}$ is $1-2 \tau$, which is in general is not in $\Omega^{N}=\left\{x_{1}<x_{2}<x_{3}<\ldots<x_{N-1}\right\}$, so we use a piecewise linear interpolant of the previous iterate to determine $\mathbf{Y}_{r}^{[k+1]}(1-2 \tau)$. Now, using our inductive argument, we have

$$
\begin{aligned}
\left|\left(\mathbf{Y}_{r}^{[k+1]}-\boldsymbol{y}\right)(1-2 \tau)\right| & =\left|\left(\overline{\mathbf{Y}}^{[k]}-\boldsymbol{y}\right)(1-2 \tau)\right|=\left|\left(\mathbf{Y}^{[k]}-\boldsymbol{y}\right)(1-2 \tau)\right| \\
& \leq\left|\left(\mathbf{Y}^{[k]}-\overline{\boldsymbol{y}}\right)(1-2 \tau)\right|+|(\overline{\boldsymbol{y}}-\boldsymbol{y})(1-2 \tau)|, \quad(5.3
\end{aligned}
$$


where $\overline{\boldsymbol{y}}$ is the piecewise linear interpolant of $\boldsymbol{y}$ using grid points of $\bar{\Omega}_{c}^{N}$. For the second term on the right-hand side of (5.3), using solution decomposition $\boldsymbol{y}$ as in (3.1), we get

$$
|(\overline{\boldsymbol{y}}-\boldsymbol{y})(1-2 \tau)| \leq|(\overline{\boldsymbol{v}}-\boldsymbol{v})(1-2 \tau)|+|(\overline{\boldsymbol{w}}-\boldsymbol{w})(1-2 \tau)| .
$$

Note that $(1-2 \tau)$ lies in $\bar{\Omega}_{c}$. For any $\mathbf{z} \in C^{2}\left(\bar{\Omega}_{c}\right)$, standard argument of piecewise linear interpolant $\overline{\mathbf{z}}$ gives

$$
|(\mathbf{z}-\overline{\mathbf{z}})(1-2 \tau)| \leq C h_{c}^{2}\left\|\mathbf{z}^{(2)}\right\|_{\bar{\Omega}_{c}} \quad \text { and } \quad|(\mathbf{z}-\overline{\mathbf{z}})(1-2 \tau)| \leq C\|\mathbf{z}\|_{\bar{\Omega}_{c}} .
$$

For the first term on the right-hand side of (5.4), we use the first bound of (5.5), $h_{c} \leq C N^{-1}$, and Lemma 2 to get

$$
|(\overline{\boldsymbol{v}}-\boldsymbol{v})(1-2 \tau)| \leq C h_{c}^{2}\left\|\boldsymbol{v}^{(2)}\right\|_{\bar{\Omega}_{c}} \leq C N^{-2} .
$$

For the second term on the right-hand side of (5.4), when $\tau=\frac{4 \varepsilon}{\alpha} \ln N$, note that the layer function $\boldsymbol{w}$ is monotonically increasing in the region $(1 / 3,1-\tau) \subset \bar{\Omega}_{c}$. Hence using the second bound of (5.5), we have

$$
|(\overline{\boldsymbol{w}}-\boldsymbol{w})(1-2 \tau)| \leq C\|\boldsymbol{w}\|_{\bar{\Omega}_{c}} .
$$

Now, using (5.6) in (5.3) we have

$$
\begin{aligned}
\left|\left(\mathbf{Y}_{r}^{[k+1]}-\boldsymbol{y}\right)(1-2 \tau)\right| & \leq C 2^{-k}+C N^{-2} \ln ^{3} N+C N^{-2} \\
& \leq C 2^{-k}+C N^{-2} \ln ^{3} N .
\end{aligned}
$$

Consider the mesh function

$$
\begin{aligned}
\Psi^{ \pm}\left(x_{i}\right)= & \mathbf{C}\left(\frac{3+x_{i}}{2}\right) 2^{-k}+\mathbf{C}\left(1+x_{i}\right) N^{-2} \ln ^{3} N \\
& +\mathbf{C}\left(x_{i}-(1-2 \tau)\right) \varepsilon^{-1} N^{-2} \ln ^{2} N \pm\left(\mathbf{Y}_{r}^{[k+1]}-\boldsymbol{y}\right)\left(x_{i}\right),
\end{aligned}
$$

where $\mathbf{C}$ is positive constants suitably chosen so that the following are satisfied.

Note that, $\Psi^{ \pm}(1-2 \tau)>0, \Psi^{ \pm}(1)>0$ and $L^{N} \Psi^{ \pm}\left(x_{i}\right)>0$. Using the discrete maximum principle for the operator $L^{N}$ on $\bar{\Omega}_{r}^{N}$ we get,

$$
\begin{aligned}
\left\|\left(\mathbf{Y}_{r}^{[k+1]}-\boldsymbol{y}\right)\right\|_{\bar{\Omega}_{r}^{N}} \leq & C\left(\frac{3+x_{i}}{2}\right) 2^{-k}+C\left(1+x_{i}\right) N^{-2} \ln ^{3} N \\
& +C\left(x_{i}-(1-2 \tau)\right) \varepsilon^{-1} N^{-2} \ln ^{2} N .
\end{aligned}
$$

Consequently,

$$
\begin{aligned}
\left\|\left(\mathbf{Y}_{r}^{[k+1]}-\boldsymbol{y}\right)\right\|_{\bar{\Omega}_{r}^{N} \backslash \bar{\Omega}_{c}} & \leq 4 C\left(\frac{1}{2}\right) 2^{-k}+2 C N^{-2} \ln ^{3} N+2 C \tau \varepsilon^{-1} N^{-2} \ln ^{2} N \\
& \leq C 2^{-(k+1)}++C N^{-2} \ln ^{3} N+C \tau \varepsilon^{-1} N^{-2} \ln ^{2} N .
\end{aligned}
$$

But since $\tau=\frac{4 \varepsilon}{\alpha} \ln N$, this gives

$$
\left\|\left(\mathbf{Y}_{r}^{[k+1]}-\boldsymbol{y}\right)\right\|_{\bar{\Omega}_{r}^{N} \backslash \bar{\Omega}_{c}} \leq C 2^{-(k+1)}+C N^{-2} \ln ^{3} N .
$$


Case (ii): Error bound estimation on $\bar{\Omega}_{c}^{N}$. We use solution decomposition as in Lemma 2 at each point $x_{i} \in \bar{\Omega}_{c}^{N}$, the difference $\left(\mathbf{Y}_{c}^{[k+1]}-\boldsymbol{y}\right)$ can be written in the form

$$
\left(\mathbf{Y}_{c}^{[k+1]}-\boldsymbol{y}\right)\left(x_{i}\right)=\left(\mathbf{V}_{c}^{[k+1]}-\boldsymbol{v}\right)\left(x_{i}\right)+\left(\mathbf{W}_{c}^{[k+1]}-\boldsymbol{w}\right)\left(x_{i}\right) .
$$

Suppose that $(1-\tau)$ lies in $\bar{\Omega}_{r}$. For any $\mathbf{z} \in \mathbf{C}^{2}\left(\bar{\Omega}_{r}\right)$, standard argument of piecewise linear interpolant $\overline{\mathbf{z}}$ gives

$$
|(\mathbf{z}-\overline{\mathbf{z}})(1-\tau)| \leq C h_{r}^{2}\left\|\mathbf{z}^{(2)}\right\|_{\bar{\Omega}_{r}} .
$$

In the proposed scheme we use the mid-point difference scheme on $\bar{\Omega}_{c}^{N}$. One can deduce the following truncation error estimate as in [12] on $x_{i} \in \bar{\Omega}_{c}^{N}$ as

$$
\left\|\left(\mathbf{L}^{N}-\mathbf{L}\right) \boldsymbol{y}\right\|_{\Omega_{c}^{N}} \leq\left(\begin{array}{c}
C h_{c}^{2}\left\|y_{1}^{(4)}\right\|_{\Omega_{c}^{N}}+C h_{c}^{2}\left\|y_{2}^{(2)}\right\|_{\Omega_{c}^{N}} \\
C \varepsilon h_{c}^{2}\left\|y_{2}^{(4)}\right\|_{\Omega_{c}^{N}}+C h_{c}^{2}\left(\left\|y_{2}^{(3)}\right\|_{\Omega_{c}^{N}}+\left\|y_{1}^{(2)}\right\|_{\Omega_{c}^{N}}\right)
\end{array}\right) .
$$

Subcase (i): For the first term on the right-hand side of (5.8), using the above local truncation error estimate, $h_{c} \leq C N^{-1}, \varepsilon \leq C N^{-1}$ and Lemma 2, we get

$$
\begin{aligned}
& \left\|\mathbf{L}^{N}\left(\mathbf{V}_{c}^{(k+1)}-\boldsymbol{v}\right)\right\|_{\Omega_{c}^{N}}=\|\mathbf{f}-\mathbf{L} \boldsymbol{v}\|_{\Omega_{c}^{N}}=\left\|\left(\mathbf{L}^{N}-\mathbf{L}\right) \boldsymbol{v}\right\|_{\Omega_{c}^{N}} \\
& \quad \leq\left(\begin{array}{c}
C h_{c}^{2}\left\|v_{1}^{(4)}\right\|_{\Omega_{c}^{N}}+C h_{c}^{2}\left\|v_{2}^{(2)}\right\|_{\Omega_{c}^{N}} \\
C \varepsilon h_{c}^{2}\left\|v_{2}^{(4)}\right\|_{\Omega_{c}^{N}}+C h_{c}^{2}\left(\left\|v_{2}^{(3)}\right\|_{\Omega_{c}^{N}}+\left\|v_{1}^{(2)}\right\|_{\Omega_{c}^{N}}\right)
\end{array}\right) \leq\left(\begin{array}{l}
C N^{-2} \\
C N^{-2}
\end{array}\right) \leq C N^{-2} .
\end{aligned}
$$

Now, using our inductive argument, the bound of (5.9), $h_{r} \leq C N^{-1}, \varepsilon \leq$ $C N^{-1}$, and Lemma 2, we get

$$
\begin{aligned}
\left|\left(\mathbf{V}_{c}^{[k+1]}-\boldsymbol{v}\right)(1-\tau)\right| & =\left|\left(\overline{\mathbf{V}}_{r}^{[k+1]}-\boldsymbol{v}\right)(1-\tau)\right|=|(\overline{\mathbf{V}}-\boldsymbol{v})(1-\tau)| \\
& \leq C h_{r}^{2}\left\|\boldsymbol{v}^{(2)}\right\|_{\bar{\Omega}_{r}} \leq C N^{-2}
\end{aligned}
$$

where we have used the fact that $(1-\tau)$ is the mesh point of $\bar{\Omega}_{r}^{N}$.

Consider the mesh function

$$
\Phi^{ \pm}\left(x_{i}\right)=\mathbf{C}\left(\frac{x_{i}}{2(1-\tau)}\right) 2^{-k}+\left(1+x_{i}\right) \mathbf{C} N^{-2} \pm\left(\mathbf{V}_{c}^{[k+1]}-\boldsymbol{v}\right)\left(x_{i}\right),
$$

where $C$ is positive constants to be choosen suitably, so that the following expressions are satisfied. Note that $\Phi^{ \pm}(0)>0, \Phi^{ \pm}(1-\tau)>0, L^{N} \Phi^{ \pm}\left(x_{i}\right)>0$.

We use the discrete maximum principle for the operator $L^{N}$ on $\bar{\Omega}_{c}^{N}$ to get

$$
\begin{aligned}
\left\|\mathbf{V}_{c}^{[k+1]}-\boldsymbol{v}\right\|_{\bar{\Omega}_{c}^{N}} & \leq C\left(\frac{1}{2}\right) 2^{-k}+C(2-\tau) N^{-2} \\
& \leq C 2^{-(k+1)}+C N^{-2} .
\end{aligned}
$$

Subcase (ii): For the second term on the right-hand side of (5.8), when $\tau=\frac{4 \varepsilon}{\alpha} \ln N$, using the arguments discussed as in ([11], Lemma 6) for $x_{i} \in \Omega_{c}^{N}$ we get

$$
\left\|\mathbf{W}_{c}^{[k+1]}-\boldsymbol{w}\right\|_{\Omega_{c}^{N}} \leq C N^{-2}
$$


Now, using error bound for the smooth and layer parts we get

$$
\left\|\left(\mathbf{Y}_{c}^{[k+1]}-\boldsymbol{y}\right)\right\|_{\Omega_{c}^{N}} \leq C 2^{-(k+1)}+C N^{-2} \ln ^{3} N .
$$

Combining the error bounds (5.7) and (5.10), we have

$$
\left\|\mathbf{Y}^{[k+1]}-\boldsymbol{y}\right\|_{\bar{\Omega}^{N}} \leq C 2^{-(k+1)}+C N^{-2} \ln ^{3} N .
$$

This completes the proof.

Now we will show that the discrete Schwarz iterates converge at a higher rate than that suggested by Lemma 5 .

Lemma 6. Let $\boldsymbol{Y}^{[k]}\left(x_{i}\right)$ be the $k^{\text {th }}$ iterate of the discrete Schwarz method described in Section 4. Then there exists some $C$ such that

$$
\left\|\boldsymbol{Y}^{[k+1]}-\boldsymbol{Y}^{[k]}\right\|_{\bar{\Omega}^{N}} \leq C \nu^{k}, \text { where } \nu=\left(1+\frac{\tau \alpha}{2 \varepsilon N}\right)^{-N / 2}<1 .
$$

Furthermore, if $\tau=\frac{4 \varepsilon}{\alpha} \ln N$, then $\nu \leq 2 N^{-1}$.

Proof. At the first iteration $\left\|\mathbf{Y}^{[0]}\right\|_{\Omega^{N}}=\mathbf{0}$. Then clearly

$$
\left\|\mathbf{Y}^{[1]}-\mathbf{Y}^{[0]}\right\|_{\Omega^{N}}=\left\|\mathbf{Y}^{[1]}\right\|_{\Omega^{N}}
$$

$\mathbf{Y}_{r}^{[1]}$ satisfies

$$
\begin{aligned}
& \mathbf{L}^{N} \mathbf{Y}_{r}^{[1]}=\mathbf{f}_{i} \quad \text { for } \quad x_{i} \in \Omega_{r}^{N}, \\
& \mathbf{Y}_{r}^{[1]}(1-2 \tau)=\overline{\mathbf{Y}}^{[0]}(1-2 \tau), \quad \mathbf{Y}_{r}^{[1]}(1)=\boldsymbol{y}(1)
\end{aligned}
$$

Therefore, we use Lemma 4 to obtain $\left\|\mathbf{Y}_{r}^{[1]}\right\|_{\bar{\Omega}_{r}^{N}} \leq C$.

Consequently, $\left\|\mathbf{Y}_{r}^{[1]}\right\|_{\bar{\Omega}_{r}^{N} \backslash \bar{\Omega}_{c}} \leq C$. Also $\mathbf{Y}_{c}^{[1]}$ satisfies

$$
\begin{aligned}
& \mathbf{L}^{N} \mathbf{Y}_{c}^{[1]}=\mathbf{f}_{i-1 / 2} \text { for } x_{i} \in \Omega_{c}^{N}, \\
& \mathbf{Y}_{c}^{[1]}(0)=\boldsymbol{y}(0), \quad \mathbf{Y}_{c}^{[1]}(1-\tau)=\overline{\mathbf{Y}}_{r}^{[1]}(1-\tau) .
\end{aligned}
$$

Therefore, we can apply Lemma 4 to get $\left\|\mathbf{Y}_{c}^{[1]}\right\|_{\bar{\Omega}_{c}^{N}} \leq C$. Combining all these estimates we obtain

$$
\left\|\mathbf{Y}^{[1]}-\mathbf{Y}^{[0]}\right\|_{\bar{\Omega}^{N}} \leq C \nu^{0} .
$$

Thus, the result holds for $k=0$ and the proof is now completed by induction argument. Assume that for an arbitrary integer $k \geq 0$

$$
\left\|\mathbf{Y}^{[k+1]}-\mathbf{Y}^{[k]}\right\|_{\bar{\Omega}^{N}} \leq \mathbf{C} \nu^{k}, \text { where } \nu=\left(1+\frac{\alpha \tau}{2 \varepsilon N}\right)^{-N / 2}
$$

Note that $\mathbf{L}^{N}\left(\mathbf{Y}_{c}^{[k+1]}-\mathbf{Y}_{c}^{[k]}\right)\left(x_{i}\right)=\mathbf{0}$ for $x_{i} \in \Omega_{c}^{N},\left(\mathbf{Y}_{c}^{[k+1]}-\mathbf{Y}_{c}^{[k]}\right)(0)=\mathbf{0}$, and $\left|\left(\mathbf{Y}_{c}^{[k+1]}-\mathbf{Y}_{c}^{[k]}\right)(1-\tau)\right| \leq C \nu^{k}$. 
Let $\Phi_{c}^{[k+1]}\left(x_{i}\right)=\left(\Phi_{1, c}^{[k+1]}\left(x_{i}\right), \Phi_{2, c}^{[k+1]}\left(x_{i}\right)\right)^{T}$ be the solution of

$$
\left\{\begin{array}{l}
A \delta^{2} \Phi_{c}^{[k+1]}\left(x_{i}\right)+\alpha D^{-} \Phi_{c}^{[k+1]}\left(x_{i}\right)+\beta \hat{\Phi}_{c}^{[k+1]}\left(x_{i}\right)=\mathbf{0} \quad \text { for } \quad x_{i} \in \Omega_{c}^{N}, \\
\Phi_{c}^{[k+1]}\left(x_{0}\right)=\mathbf{0}, \quad \Phi_{c}^{[k+1]}\left(x_{n}\right)=\mathbf{C} \nu^{k}
\end{array}\right.
$$

where $A=\left(\begin{array}{cc}-1 & 0 \\ 0 & -\varepsilon\end{array}\right)$. Using the maximum principle argument we note that $\Phi_{c}^{[k+1]}(0) \geq \mathbf{0}, \Phi_{c}^{[k+1]}(1-\tau) \geq \mathbf{0}, \Phi_{c}^{[k+1]}\left(x_{i}\right) \geq \mathbf{0}$ for $x_{i} \in \bar{\Omega}_{c}^{N}$, and thus one can easily deduce that $L^{N} \Phi_{c}^{[k+1]}\left(x_{i}\right) \geq \mathbf{0}$, for $x_{i} \in \Omega_{c}^{N}$. Hence

$$
\begin{gathered}
L^{N}\left(\Phi_{c}^{[k+1]}-\left(\mathbf{Y}_{c}^{[k+1]}-\mathbf{Y}_{c}^{[k]}\right)\right)\left(x_{i}\right)=L^{N}\left(\Phi_{c}^{[k+1]}\right)\left(x_{i}\right)-L^{N}\left(\mathbf{Y}_{c}^{[k+1]}-\mathbf{Y}_{c}^{[k]}\right)\left(x_{i}\right), \\
\geq \mathbf{0}, \text { as } L^{N}\left(\mathbf{Y}_{c}^{[k+1]}-\mathbf{Y}_{c}^{[k]}\right)\left(x_{i}\right)=\mathbf{0} \text { for } x_{i} \in \Omega_{c}^{N}, \\
\Phi_{c}^{[k+1]}(0)-\left(\mathbf{Y}_{c}^{[k+1]}-\mathbf{Y}_{c}^{[k]}\right)(0) \geq \mathbf{0}, \Phi_{c}^{[k+1]}(1-\tau)-\left(\mathbf{Y}_{c}^{[k+1]}-\mathbf{Y}_{c}^{[k]}\right)(1-\tau) \geq \mathbf{0} .
\end{gathered}
$$

Then by using Lemma 3 we have

$$
\left(\mathbf{Y}_{c}^{[k+1]}-\mathbf{Y}_{c}^{[k]}\right)\left(x_{i}\right) \leq \Phi_{c}^{[k+1]}\left(x_{i}\right) \text { for } \quad x_{i} \in \Omega_{c}^{N} .
$$

The exact solution to the difference problem (5.11) is

$$
\Phi_{c}^{[k+1]}\left(x_{i}\right)=\mathbf{C} \nu^{k}\left(m_{1}^{i}-m_{2}^{i}\right) /\left(m_{1}^{N}-m_{2}^{N}\right),
$$

where

$$
\begin{aligned}
m_{1} & =\left(1+\frac{\alpha h_{c}}{2 \varepsilon}+\frac{\beta h_{c}^{2}}{4 \varepsilon}\right)+\sqrt{\left(1+\frac{\alpha h_{c}}{2 \varepsilon}+\frac{\beta h_{c}^{2}}{4 \varepsilon}\right)^{2}+\left(-1-\frac{\alpha h_{c}}{\varepsilon}+\frac{\beta h_{c}^{2}}{2 \varepsilon}\right)} \\
& \geq 1+\frac{\alpha h_{c}}{2 \varepsilon}=\left(1+\frac{\alpha(1-\tau)}{2 \varepsilon N}\right) \geq\left(1+\frac{\alpha \tau}{2 \varepsilon N}\right), \\
m_{2} & =\left(1+\frac{\alpha h_{c}}{2 \varepsilon}+\frac{\beta h_{c}^{2}}{4 \varepsilon}\right)-\sqrt{\left(1+\frac{\alpha h_{c}}{2 \varepsilon}+\frac{\beta h_{c}^{2}}{4 \varepsilon}\right)^{2}+\left(-1-\frac{\alpha h_{c}}{\varepsilon}+\frac{\beta h_{c}^{2}}{2 \varepsilon}\right)} .
\end{aligned}
$$

Now

$$
\mathbf{L}^{N}\left(\mathbf{Y}_{r}^{[k+2]}-\mathbf{Y}_{r}^{[k+1]}\right)\left(x_{i}\right)=\mathbf{0}, \quad \forall x_{i} \in \bar{\Omega}_{r}^{N}, \quad\left(\mathbf{Y}_{r}^{[k+2]}-\mathbf{Y}_{r}^{[k+1]}\right)(1)=\mathbf{0} .
$$

Using our inductive hypothesis and (5.12)

$$
\begin{aligned}
& \left|\left(\mathbf{Y}_{r}^{[k+2]}-\mathbf{Y}_{r}^{[k+1]}\right)(1-2 \tau)\right|=\left|\left(\overline{\mathbf{Y}}_{c}^{[k+1]}-\overline{\mathbf{Y}}_{c}^{[k]}\right)(1-2 \tau)\right| \\
& \quad=\left|\left(\mathbf{Y}_{c}^{[k+1]}-\mathbf{Y}_{c}^{[k]}\right)(1-2 \tau)\right| \leq \Phi_{c}^{[k+1]}(1-2 \tau),
\end{aligned}
$$

where we have used the fact that $(1-2 \tau)$ is the mesh point of $\bar{\Omega}_{c}^{N}$. Using Lemma 4 we obtain

$$
\left\|\mathbf{Y}_{r}^{[k+2]}-\mathbf{Y}_{r}^{[k+1]}\right\|_{\bar{\Omega}_{r}^{N}} \leq \Phi_{c}^{[k+1]}(1-2 \tau) .
$$


Here we used

$$
\begin{aligned}
& \Phi_{c}^{[k+1]}(1-2 \tau)=\mathbf{C} \nu^{k} \frac{m_{1}^{N / 2}-m_{2}^{N / 2}}{m_{1}^{N}-m_{2}^{N}} \leq \frac{\mathbf{C} \nu^{k}}{m_{1}^{N / 2}} \\
& =\mathbf{C} \nu^{k}\left(1+\frac{\tau \alpha}{2 \varepsilon N}\right)^{-N / 2}=\mathbf{C} \nu^{k}\left(1+\frac{\tau \alpha}{2 \varepsilon N}\right)^{-N / 2}=\mathbf{C} \nu^{k+1} .
\end{aligned}
$$

Therefore we get

$$
\left\|\mathbf{Y}_{r}^{[k+2]}-\mathbf{Y}_{r}^{[k+1]}\right\|_{\bar{\Omega}_{r}^{N}} \leq C \nu^{k+1}
$$

and consequently

$$
\left\|\mathbf{Y}_{r}^{[k+2]}-\mathbf{Y}_{r}^{[k+1]}\right\|_{\bar{\Omega}_{r}^{N} \backslash \bar{\Omega}_{c}} \leq C \nu^{k+1}
$$

Finally note that

$$
\mathbf{L}^{N}\left(\mathbf{Y}_{c}^{[k+2]}-\mathbf{Y}_{c}^{[k+1]}\right)\left(x_{i}\right)=\mathbf{0} \quad \text { for } \quad x_{i} \in \Omega_{c}^{N}, \quad\left(\mathbf{Y}_{c}^{[k+2]}-\mathbf{Y}_{c}^{[k+1]}\right)(0)=\mathbf{0}
$$

Using our inductive hypothesis and (5.13), we have

$$
\begin{gathered}
\left|\left(\mathbf{Y}_{c}^{[k+2]}-\mathbf{Y}_{c}^{[k+1]}\right)(1-\tau)\right|=\left|\left(\overline{\mathbf{Y}}_{r}^{[k+2]}-\overline{\mathbf{Y}}_{r}^{[k+1]}\right)(1-\tau)\right| \\
=\left|\left(\mathbf{Y}_{r}^{[k+2]}-\mathbf{Y}_{r}^{[k+1]}\right)(1-\tau)\right| \leq C \nu^{[k+1]},
\end{gathered}
$$

where we have used the fact that $(1-\tau)$ is the mesh point of $\bar{\Omega}_{c}^{N}$. Therefore, we can apply Lemma 4 to get

$$
\left\|\mathbf{Y}_{c}^{[k+2]}-\mathbf{Y}_{c}^{[k+1]}\right\|_{\bar{\Omega}_{c}^{N}} \leq C \nu^{k+1} .
$$

Combining the estimates (5.14) and (5.15) we obtain,

$$
\left\|\mathbf{Y}^{[k+2]}-\mathbf{Y}^{[k+1]}\right\|_{\bar{\Omega}^{N}} \leq C \nu^{k+1} .
$$

For $\tau=\frac{4 \varepsilon}{\alpha} \ln N$ using the arguments given in Lemma 4.1 of [10] we obtain,

$$
\nu=\left(1+\frac{\tau \alpha}{2 \varepsilon N}\right)^{-N / 2}=\left(1+\frac{2 \ln N}{N}\right)^{-N / 2} \leq 2 N^{-1}, \quad N \geq 1 .
$$

The following theorem is the main result of this paper, combining Lemmas 5 and 6 we prove that two iterations are sufficient to attain almost second-order convergence.

Theorem 2. Let $\boldsymbol{y}(x)$ be the solution to (1.6)-(1.7) and $\boldsymbol{Y}^{[k]}\left(x_{i}\right)$ be the $k^{\text {th }}$ iterate of the discrete Schwarz method described in Section 4. If $\tau=\frac{4 \varepsilon}{\alpha} \ln N$ and $N>2$, then

$$
\left\|\boldsymbol{Y}^{[k]}-\boldsymbol{y}\right\|_{\bar{\Omega}^{N}} \leq C N^{-k}+C N^{-2} \ln ^{3} N
$$


Proof. From Lemma 6 there exists $\mathbf{Y}$ such that $\mathbf{Y}:=\mathrm{lt}_{k \rightarrow \infty} \mathbf{Y}^{[k]}$. We know from Lemma 5 that there exists $C$ such that

$$
\left\|\mathbf{Y}^{[k]}-\boldsymbol{y}\right\|_{\bar{\Omega}^{N}} \leq C 2^{-k}+C N^{-2} \ln ^{3} N .
$$

This implies that

$$
\|\mathbf{Y}-\boldsymbol{y}\|_{\bar{\Omega}^{N}} \leq C N^{-2} \ln ^{3} N .
$$

Also from Lemma 6 that there exists $C$ such that

$$
\left\|\mathbf{Y}^{[k+1]}-\mathbf{Y}^{[k]}\right\|_{\bar{\Omega}^{N}} \leq C N^{-k} .
$$

Consequently, for $N \geq 2$, there exists $C$ such that

$$
\left\|\mathbf{Y}^{[k]}-\mathbf{Y}\right\|_{\bar{\Omega}^{N}} \leq C \sum_{l=k}^{\infty} N^{-l}=C\left[\frac{N^{-k}}{1-N^{-1}}\right] \leq C N^{-k} .
$$

Thus, using (5.16) and (5.17), we conclude that

$$
\begin{aligned}
& \left\|\mathbf{Y}^{[k]}-\boldsymbol{y}\right\|_{\bar{\Omega}^{N}}=\left\|\mathbf{Y}^{[k]}-\mathbf{Y}+\mathbf{Y}-\boldsymbol{y}\right\|_{\bar{\Omega}^{N}} \\
& \quad \leq\left\|\mathbf{Y}^{[k]}-\mathbf{Y}\right\|_{\bar{\Omega}^{N}}+\|\mathbf{Y}-\boldsymbol{y}\|_{\bar{\Omega}^{N}} \leq C N^{-k}+C N^{-2} \ln ^{3} N .
\end{aligned}
$$

\section{$6 \quad$ Numerical experiments}

In this section, we consider one example to illustrate the theoretical results for the BVPs (1.1)-(1.2). The stopping criterion for the iterative procedure is taken to be

$$
\left\|\mathbf{Y}^{[k+1]}-\mathbf{Y}^{[k]}\right\|_{\bar{\Omega}^{N}} \leq 10^{-14} \text {, for } j=1,2 .
$$

We normally omit the superscript $k$ on the final Schwarz iterate and write simply $Y_{j}^{N}$. Let $Y_{j}^{N}$ be a Schwarz numerical approximation for the exact solution $y_{j}$ on the mesh $\Omega^{N}$ and $N$ is the number of mesh points. For a finite set of values of $\varepsilon=\left\{2^{0}, \ldots, 2^{-30}\right\}$, we compute the maximum point-wise two mesh difference errors for $j=1,2$

$$
\left\|Y_{j}^{N}-y_{j}\right\|_{\Omega^{N}} \approx D_{\varepsilon, j}^{N}:=\left\|Y_{j}^{N}-\bar{Y}_{j}^{2 N}\right\|_{\Omega^{N}}, \quad D_{j}^{N}=\max _{\varepsilon} D_{\varepsilon, j}^{N},
$$

where $\bar{Y}_{j}^{2 N}$ is the numerical solution obtained on a mesh with the same transition points, but with $2 N$ intervals in each subdomain. From these quantities the $\varepsilon$-uniform order of convergence is computed from

$$
p_{j}^{N}=\log _{2}\left\{D_{j}^{N} / D_{j}^{2 N}\right\} \text {, for } j=1,2 .
$$

The computed maximum pointwise errors $D_{j}^{N},(j=1,2)$ and the computed order of convergence $p_{j}^{N},(j=1,2)$ and $k$ (the number of iterations computed) for various values of $N$ and $\varepsilon$ are tabulated in Table 1 and Table 2. The nodal errors are plotted as graphs in Figure 1. We can see that the errors decrease as $N$ increases. The computed rates of convergence are almost second-order, with the usual $\ln N$ factor associated with these techniques. 
Example 1. Consider the BVP

$$
\begin{array}{r}
-\varepsilon y^{i v}(x)+(2-x) y^{\prime \prime \prime}(x)+(1+x) y^{\prime \prime}(x)-\left(x^{2} / 5\right) y(x)=-\sinh x, \\
y(0)=0, \quad y^{\prime \prime}(0)=0, \quad y(1)=0, \quad y^{\prime \prime}(1)=0 .
\end{array}
$$

The numerical results are presented in Table 1 and Table 2.

Table 1. Values of $D_{1}^{N}, p_{1}^{N}$ for the solution component $Y_{1}$ for the Example 1

\begin{tabular}{cccccc}
\hline \multicolumn{5}{c}{ Number of mesh points $\mathrm{N}$} \\
\hline & 64 & 128 & 256 & 512 & 1024 \\
\hline $2^{0}$ & $4.5356 \mathrm{e}-008$ & $1.1447 \mathrm{e}-008$ & $2.8752 \mathrm{e}-009$ & $7.2046 \mathrm{e}-010$ & $1.8032 \mathrm{e}-010$ \\
$2^{-2}$ & $1.7836 \mathrm{e}-007$ & $4.4157 \mathrm{e}-008$ & $1.0975 \mathrm{e}-008$ & $2.7349 \mathrm{e}-009$ & $6.8258 \mathrm{e}-010$ \\
$2^{-4}$ & $2.3850 \mathrm{e}-007$ & $5.8368 \mathrm{e}-008$ & $1.4400 \mathrm{e}-008$ & $3.5733 \mathrm{e}-009$ & $8.8980 \mathrm{e}-010$ \\
$2^{-6}$ & $5.5922 \mathrm{e}-007$ & $7.3269 \mathrm{e}-008$ & $1.0758 \mathrm{e}-008$ & $2.6651 \mathrm{e}-009$ & $6.6270 \mathrm{e}-010$ \\
$2^{-8}$ & $3.6738 \mathrm{e}-006$ & $8.3723 \mathrm{e}-007$ & $1.9091 \mathrm{e}-007$ & $4.3482 \mathrm{e}-008$ & $9.8857 \mathrm{e}-009$ \\
$2^{-10}$ & $5.1796 \mathrm{e}-006$ & $1.2608 \mathrm{e}-006$ & $3.0798 \mathrm{e}-007$ & $7.5364 \mathrm{e}-008$ & $1.8455 \mathrm{e}-008$ \\
$2^{-12}$ & $5.6146 \mathrm{e}-006$ & $1.3864 \mathrm{e}-006$ & $3.4362 \mathrm{e}-007$ & $8.5332 \mathrm{e}-008$ & $2.1212 \mathrm{e}-008$ \\
$2^{-14}$ & $5.7272 \mathrm{e}-006$ & $1.4191 \mathrm{e}-006$ & $3.5296 \mathrm{e}-007$ & $8.7960 \mathrm{e}-008$ & $2.1942 \mathrm{e}-008$ \\
$2^{-16}$ & $5.7556 \mathrm{e}-006$ & $1.4274 \mathrm{e}-006$ & $3.5532 \mathrm{e}-007$ & $8.8625 \mathrm{e}-008$ & $2.2128 \mathrm{e}-008$ \\
$2^{-18}$ & $5.7627 \mathrm{e}-006$ & $1.4294 \mathrm{e}-006$ & $3.5591 \mathrm{e}-007$ & $8.8792 \mathrm{e}-008$ & $2.2174 \mathrm{e}-008$ \\
$2^{-20}$ & $5.7645 \mathrm{e}-006$ & $1.4299 \mathrm{e}-006$ & $3.5606 \mathrm{e}-007$ & $8.8834 \mathrm{e}-008$ & $2.2186 \mathrm{e}-008$ \\
$2^{-22}$ & $5.7650 \mathrm{e}-006$ & $1.4301 \mathrm{e}-006$ & $3.5610 \mathrm{e}-007$ & $8.8845 \mathrm{e}-008$ & $2.2189 \mathrm{e}-008$ \\
$2^{-24}$ & $5.7651 \mathrm{e}-006$ & $1.4301 \mathrm{e}-006$ & $3.5610 \mathrm{e}-007$ & $8.8847 \mathrm{e}-008$ & $2.2189 \mathrm{e}-008$ \\
$2^{-26}$ & $5.7651 \mathrm{e}-006$ & $1.4301 \mathrm{e}-006$ & $3.5611 \mathrm{e}-007$ & $8.8848 \mathrm{e}-008$ & $2.2190 \mathrm{e}-008$ \\
$2^{-28}$ & $5.7651 \mathrm{e}-006$ & $1.4301 \mathrm{e}-006$ & $3.5611 \mathrm{e}-007$ & $8.8848 \mathrm{e}-008$ & $2.2190 \mathrm{e}-008$ \\
$2^{-30}$ & $5.7651 \mathrm{e}-006$ & $1.4301 \mathrm{e}-006$ & $3.5611 \mathrm{e}-007$ & $8.8848 \mathrm{e}-008$ & $2.2190 \mathrm{e}-008$ \\
$D_{1}^{N}$ & $5.7651 \mathrm{e}-006$ & $1.4301 \mathrm{e}-006$ & $3.5611 \mathrm{e}-007$ & $8.8848 \mathrm{e}-008$ & $2.2190 \mathrm{e}-008$ \\
$p_{1}^{N}$ & $\mathbf{2 . 0 1 1 2}$ & $\mathbf{2 . 0 0 5 7}$ & $\mathbf{2 . 0 0 2 9}$ & $\mathbf{2 . 0 0 1 4}$ & - \\
\hline
\end{tabular}
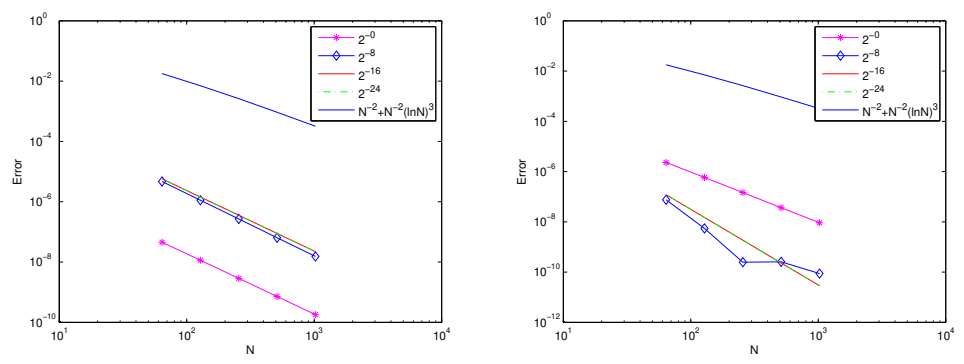

Figure 1. Nodal error for the components $Y_{1}$ and $Y_{2}$ of the Example 1

\section{$7 \quad$ Conclusions}

A singularly perturbed fourth-order ODEs of convection-diffusion problem is considered. It is shown that a designed discrete Schwarz method produces numerical approximations which converge in the maximum norm to the exact 
Table 2. Values of $D_{2}^{N}, p_{2}^{N}$ for the solution component $Y_{2}$ for the Example 1

\begin{tabular}{cccccc}
\hline \multicolumn{5}{c}{ Number of mesh points N } \\
\hline & 64 & 128 & 256 & 512 & 1024 \\
\hline $2^{0}$ & $2.3114 \mathrm{e}-006$ & $5.8302 \mathrm{e}-007$ & $1.4641 \mathrm{e}-007$ & $3.6683 \mathrm{e}-008$ & $9.1808 \mathrm{e}-009$ \\
$2^{-2}$ & $6.1032 \mathrm{e}-006$ & $1.5000 \mathrm{e}-006$ & $3.7168 \mathrm{e}-007$ & $9.2499 \mathrm{e}-008$ & $2.3072 \mathrm{e}-008$ \\
$2^{-4}$ & $5.6156 \mathrm{e}-008$ & $5.2490 \mathrm{e}-009$ & $3.5293 \mathrm{e}-009$ & $1.1476 \mathrm{e}-009$ & $3.1933 \mathrm{e}-009$ \\
$2^{-6}$ & $6.5589 \mathrm{e}-008$ & $1.9600 \mathrm{e}-008$ & $5.0806 \mathrm{e}-009$ & $1.2941 \mathrm{e}-009$ & $3.2651 \mathrm{e}-010$ \\
$2^{-8}$ & $6.0556 \mathrm{e}-008$ & $3.5318 \mathrm{e}-009$ & $4.7276 \mathrm{e}-010$ & $2.7546 \mathrm{e}-010$ & $8.6772 \mathrm{e}-011$ \\
$2^{-10}$ & $1.0437 \mathrm{e}-007$ & $1.1904 \mathrm{e}-008$ & $1.2359 \mathrm{e}-009$ & $9.5007 \mathrm{e}-011$ & $2.5776 \mathrm{e}-012$ \\
$2^{-12}$ & $1.1620 \mathrm{e}-007$ & $1.4187 \mathrm{e}-008$ & $1.7060 \mathrm{e}-009$ & $1.9786 \mathrm{e}-010$ & $2.1020 \mathrm{e}-011$ \\
$2^{-14}$ & $1.1921 \mathrm{e}-007$ & $1.4769 \mathrm{e}-008$ & $1.8263 \mathrm{e}-009$ & $2.2423 \mathrm{e}-010$ & $2.7083 \mathrm{e}-011$ \\
$2^{-16}$ & $1.1997 \mathrm{e}-007$ & $1.4916 \mathrm{e}-008$ & $1.8565 \mathrm{e}-009$ & $2.3086 \mathrm{e}-010$ & $2.8610 \mathrm{e}-011$ \\
$2^{-18}$ & $1.2016 \mathrm{e}-007$ & $1.4953 \mathrm{e}-008$ & $1.8641 \mathrm{e}-009$ & $2.3253 \mathrm{e}-010$ & $2.8992 \mathrm{e}-011$ \\
$2^{-20}$ & $1.2021 \mathrm{e}-007$ & $1.4962 \mathrm{e}-008$ & $1.8660 \mathrm{e}-009$ & $2.3294 \mathrm{e}-010$ & $2.9088 \mathrm{e}-011$ \\
$2^{-22}$ & $1.2022 \mathrm{e}-007$ & $1.4964 \mathrm{e}-008$ & $1.8665 \mathrm{e}-009$ & $2.3305 \mathrm{e}-010$ & $2.9111 \mathrm{e}-011$ \\
$2^{-24}$ & $1.2022 \mathrm{e}-007$ & $1.4965 \mathrm{e}-008$ & $1.8666 \mathrm{e}-009$ & $2.3307 \mathrm{e}-010$ & $2.9117 \mathrm{e}-011$ \\
$2^{-26}$ & $1.2022 \mathrm{e}-007$ & $1.4965 \mathrm{e}-008$ & $1.8666 \mathrm{e}-009$ & $2.3308 \mathrm{e}-010$ & $2.9119 \mathrm{e}-011$ \\
$2^{-28}$ & $1.2022 \mathrm{e}-007$ & $1.4965 \mathrm{e}-008$ & $1.8666 \mathrm{e}-009$ & $2.3308 \mathrm{e}-010$ & $2.9119 \mathrm{e}-011$ \\
$2^{-30}$ & $1.2022 \mathrm{e}-007$ & $1.4965 \mathrm{e}-008$ & $1.8666 \mathrm{e}-009$ & $2.3308 \mathrm{e}-010$ & $2.9119 \mathrm{e}-011$ \\
$D_{2}^{N}$ & $6.1032 \mathrm{e}-006$ & $1.5000 \mathrm{e}-006$ & $3.7168 \mathrm{e}-007$ & $9.2499 \mathrm{e}-008$ & $2.3072 \mathrm{e}-008$ \\
$p_{2}^{N}$ & $\mathbf{2 . 0 2 4 6}$ & $\mathbf{2 . 0 1 2 8}$ & $\mathbf{2 . 0 0 6 6}$ & $\mathbf{2 . 0 0 3 3}$ & - \\
\hline
\end{tabular}

solution. This convergence is shown to be of almost second-order. Note that from Theorem 2 , for $k \geq 2$ the $N^{-2}+N^{-2} \ln ^{3} N$ term dominates the error bound. Thus, two iterations are sufficient to attained the desired accuracy.

The present method gives improved numerical results with regard to error and order compared with the other method in $[2,15,16,17]$. From Theorem 2 it can be easily identified in which iterations, the Schwarz iterate terminates. From the given example number of iterations taken by this method is not more than two which is very much reduced when comparing iteration counts presented in $[8,9]$. This illustrates the efficiency of the method used with proposed scheme in this paper.

Numerical experiment validate the theoretical result. The graphs plotted in the figure is convergent curves in the maximum norm at nodal points for the different values of $\varepsilon$ and $N$ for the example considered. This graph clearly indicate that the optimal error bound is of order $O\left(N^{-k}+N^{-2} \ln ^{3} N\right)$ as predicted.

\section{References}

[1] M. Chandru and V. Shanthi. A Schwarz method for fourth-order singularly perturbed reaction-diffusion problem with discontinuous source term. J. Appl. Math. and Informatics, 34(5-6):495-508, 2016. https://doi.org/10.14317/jami.2016.495.

[2] J. Christy Roja and A. Tamilselvan. Numerical method for singularly perturbed fourth-order ordinary differential equations of convection-diffusion type. Journal of Mathematical Modeling, 4(1):79-102, 2016. 
[3] J. Christy Roja and A. Tamilselvan. Schwarz method for singularly perturbed second-order convection-diffusion equations. J. Appl. Math. and Informatics, 36(3-4):181-203, 2018.

[4] M.K. Kadalbajoo and Y.N. Reddy. A brief survey on numerical methods for solving singularly perturbed problems. Appl. Math. Comput., 2010. https://doi.org/10.1016/j.amc.2010.09.059.

[5] M. Feckan. Singularly perturbed higher-order boundary value problems. $J$. Differ. Equ., 3:79-102, 1994. https://doi.org/10.1006/jdeq.1994.1076.

[6] H. MacMullan, J.J.H. Miller, E. O'Riordan and G.I. Shishkin. A second-order parameter-uniform overlapping Schwarz method for reaction-diffusion problems with boundary layers. J. Comput. Appl. Math., 130(1-2):231-244, 2001. https://doi.org/10.1016/S0377-0427(99)00380-5.

[7] H. MacMullan, J.J.H. Miller, E. O'Riordan, G.I. Shishkin and S. Wang. A parameter-uniform Schwarz method for a singularly perturbed reactiondiffusion problem with an interrior layer. Appl. Num. Math., 35:323-337, 2000. https://doi.org/10.1016/S0168-9274(99)00140-3.

[8] H. MacMullen, J.J.H. Miller, E. O'Riordan and G.I. Shishkin. Schwarz iterative method for convection-diffusion problems with boundary layers. In L.G. Vulkov, J.J.H. Miller and G.I. Shishkin(Eds.), Analytical and numerical methods for convection-dominated and singularly perturbed problems, pp. 213-218. Nova Science Publishers, New York, 2000.

[9] H. MacMullen, E. O'Riordan and G.I. Shishkin. The convergence of classical Schwarz methods applied to convection-diffusion problems with regular boundary layers. Appl. Num. Math., 43:297-313, 2002. https://doi.org/10.1016/S0168-9274(01)00177-5.

[10] J.J.H. Miller, E. O'Riordan and G.I. Shishkin. Fitted numerical methods for singular perturbation problems, Error estimates in the maximum norm for linear problems in one and two dimensions. World Scientific, Singapore, 1996. https://doi.org/10.1142/2933.

[11] R. Mythili Priyadharshini, N. Ramanujam and V. Shanthi. Approximation of derivative in a system of singularly perturbed convectiondiffusion equations. J. Appl. Math. Comput., 30:369-383, 2009. https://doi.org/10.1007/s12190-008-0178-5.

[12] R. Mythili Priyadharshini, N. Ramanujam and A. Tamilselvan. Hybrid difference schemes for a system of singularly perturbed convection-diffusion equations. $J$. Appl. Math. and Informatics, 27(5-6):1001-1015, 2009.

[13] S.C.S. Rao and S. Kumar. An almost fourth-order uniformly convergent domain decomposition method for a coupled system of singularly perturbed reaction diffusion equations. J. Comput. and Appl. Math., 235:3342-3354, 2011. https://doi.org/10.1016/j.cam.2011.01.047.

[14] H.G. Roos, M. Stynes and L. Tobiska. Numerical methods for singularly perturbed differential equations, Convection-diffusion and flow problems. Springer-Verlag, 1996. https://doi.org/10.1007/978-3-662-03206-0.

[15] V. Shanthi and N. Ramanujam. Asymptotic numerical method for boundary value problems for singularly perturbed fourth-order ordinary differential equations with a weak interior layer. Appl. Math. Comput., 133:559-579, 2002. https://doi.org/10.1016/S0096-3003(01)00257-0. 
[16] V. Shanthi and N. Ramanujam. A boundary value technique for boundary value problems for singularly perturbed fourth-order ordinary differential equations. Comput. Math. Appl., 47:1673-1688, 2004. https://doi.org/10.1016/j.camwa.2004.06.015.

[17] V. Shanthi and N. Ramanujam. Computational methods for reaction-diffusion problems for fourth-order ordinary differentional equations with a small parameter at the highest derivative. Appl. Math. Comput., 147:97-113, 2004. https://doi.org/10.1016/S0096-3003(02)00654-9.

[18] M. Stephens and N. Madden. A parameter-uniform Schwarz method for a coupled system of reaction-diffusion equations. J. Comput. Appl. Math., 230:360370, 2009. https://doi.org/10.1016/j.cam.2008.12.009.

[19] M. Stynes and H.G. Roos. The midpoint upwind scheme. Appl. Numer. Math., 23(3):361-374, 1997. https://doi.org/10.1016/S0168-9274(96)00071-2.

[20] Sunil Kumar and Mukesh Kumar. An analysis of overlapping domain decomposition methods for singularly perturbed reaction-diffusion problems. J. Comput. Appl. Math., 281:250-262, 2015. https://doi.org/10.1016/j.cam.2014.12.018. 